le répétons, la législation italienne commence à envisager amélioration des jelouses de montagnes.

Nous sommes plus avancés sur ce point, mals, en principe plutôt qu'en fart, pussque les parties de notre lor de 188:, relatives à la réglementation pastorale sont, par ellesmèmes, valnes et stèrles. Et cette avance théorique, nous la perdrons peut-être bientôt, les Chambres italıennes étant acuellement sassies d'un projet de législation pastorale.

vous nous trouvons, d'ailleurs, en retard encore sur un autre point.

Fin Sardaıgne, en Basilicate, en Calabre, le gouvernement falien a institué et fortement constitué le crédit agricole, mesure fort heureuse et féconde qui, certainement, contriwera pussamment au relèvement et au développement agricole de ces diverses provinces. Mais, ce qui est nouveau et particulièrement intéressant, c'est que l'Etat dote ces cusses ou lustituts de crédat de terrains boisés ou à reboiser. or, n'est-ce pas à peu près ce que voudrait l'Association pour laniénagement des montagnes en France, lorsqu'elle deliande que les Associations diverses, même celles de crédit, les caisses de retrantes et autres, soient aptes à posséder des bols et à placer des fonds en reboisement ? Son vou, à cet igard, se trouve donc réalisé... en Italie. Pâchons qu'll se réalise aussi en France.

En terminant ce mémorre, nous tenons à nous acquitter flun agréable devoir qui est dexprmer toute notre vive oratitude à l'éminent ingéneur, chef de l'Eicole forestière Wllalıe, M. le Commandeur F. Plccioli. C'est à sa très bienseillante et gracieuse obligeance que nous devons les divers documents qui nous ont permis de rédiger cette étude ef d'y exposer les remarquables efforts faits par l'Italie pour réparer ses ruines forestières. Qu'il veuille bien, ainsi que le très dıstınguć professeur de droit à cette même Eicole, U. le Commandeur T. Pampaloni, dont nous sommes aussi Iobligé, trouver, ici, le témoignage public de notre reconmaissance.

\section{LE GAZAGE ÉLECTRIQUE DES TEXTILES}

Le gazage est une opération qui a pour but de faire disparaitre par combustion rapide et superficielle les fibrilles ou duvets dont sont recouverts les filés après les opérations de la filature. Jusqu'à ces derniers temps, cette opération s'effectuait en faisant rapidement passer les textiles dans une flamme de gaz, d'où son nom de gazage.

Mais ce made opératoire n'est pas sans présenter de nombreux inconvénients: La dépense de gaz est énorme, et l'utrlisation calorifique est si mauvaise que plus de go pour 100 de la chaleur émise est inutilisée pour le gazage et ne sert qu’à dever la température de la salle de travail. En outre, il se produit un dégagement très nuisible d'oxyde de carbone qui, malgré la ventilation, intoxique à la longue les ouvrières chargées de la surveillance des métiers à gazer. De plus, en raison des impuretés contenues dans le gaz, les fils prennent une légère coloration, ce qui n'est pas sans inconvénient pour ccux qui doivent servir à confectionner des étoffés blanches.
L'électricité, dont les applications aussi direrses que variées ne se comptent plus, est venu remédier à ces inconvénients, et, chose curieuse, c'est à un électrométallurgiste qu'est dû la résolution du problème du gazage électrique des textiles! Il est vrai que l'appareil employé est un véritable four électrique, et son inventeur, M Gustave Gin, aidé de M. Petitalot, n'a fait que transporter dans le domaine de la filature l'application de l'échauflement produit par le passage d'un courant électrique dans un conducteur métallique, si couramment utilisée en électrométallurgie.

Le principe du gazage électrique consiste à faire passer le fil dans un long tube de platine, qui est porté à l'incandescence sous l'effet du courant électıique. Ce tube, de section circulaire ou elliptique, est fendu suivantlune de ses génératrices pour laisser passer le fil.

Afin d'éviter le gauchissement du tube sous l'effet de la dilatation thermique, on utilise l'électricité propra du métal au moyen de l'artifice suivant: Aux deux extrémités, le tube est refendu une seconde fois, sur une certaine longueur, et suivant une génératrice diamétrialement opposée à la première fente. Les bandes métalliques ainsi obtenues sont rabattues et connectées à des mâchoires fixes. Mais, tandis que la connexion est simplement à angle droit pour celle du haut, cette connexion est en spirale pour celle du bas, ce qui permet au tube de se dilater librement (voir fig. 2).

Les mâchoires de connexion sont réunies aux barres d'amenée de courant par des griffes élastiquer. Les deux griffes et les deux màchoires sont portées sur une plaquesupport en marbre, ou en porcelaine, sur laquelle vient s'appuyer le carter, lequel, outre qu'll atténue le rayon nement extérieur du tube de platine, constitue, par sa forme et par sa disposition, une boite où se fait l'appel d'air nécessaire à la combustion des parties fibrilleuses et dureteuses, en même temps qu'un dispositif captant complètement les gaz et les produits de la combustion qu'évacue au dehors une ventilation aspirante énergique.

Les figures 4 et $j$ permettent de se rendre facilement compte du fonctionnement d'un brùleur muni de sa boîte d'aspiration; la fig. 4 montre deux brûleurs en élévation; la fig. 5 est une coupe verticale faite suivant l'axe du brùleur.

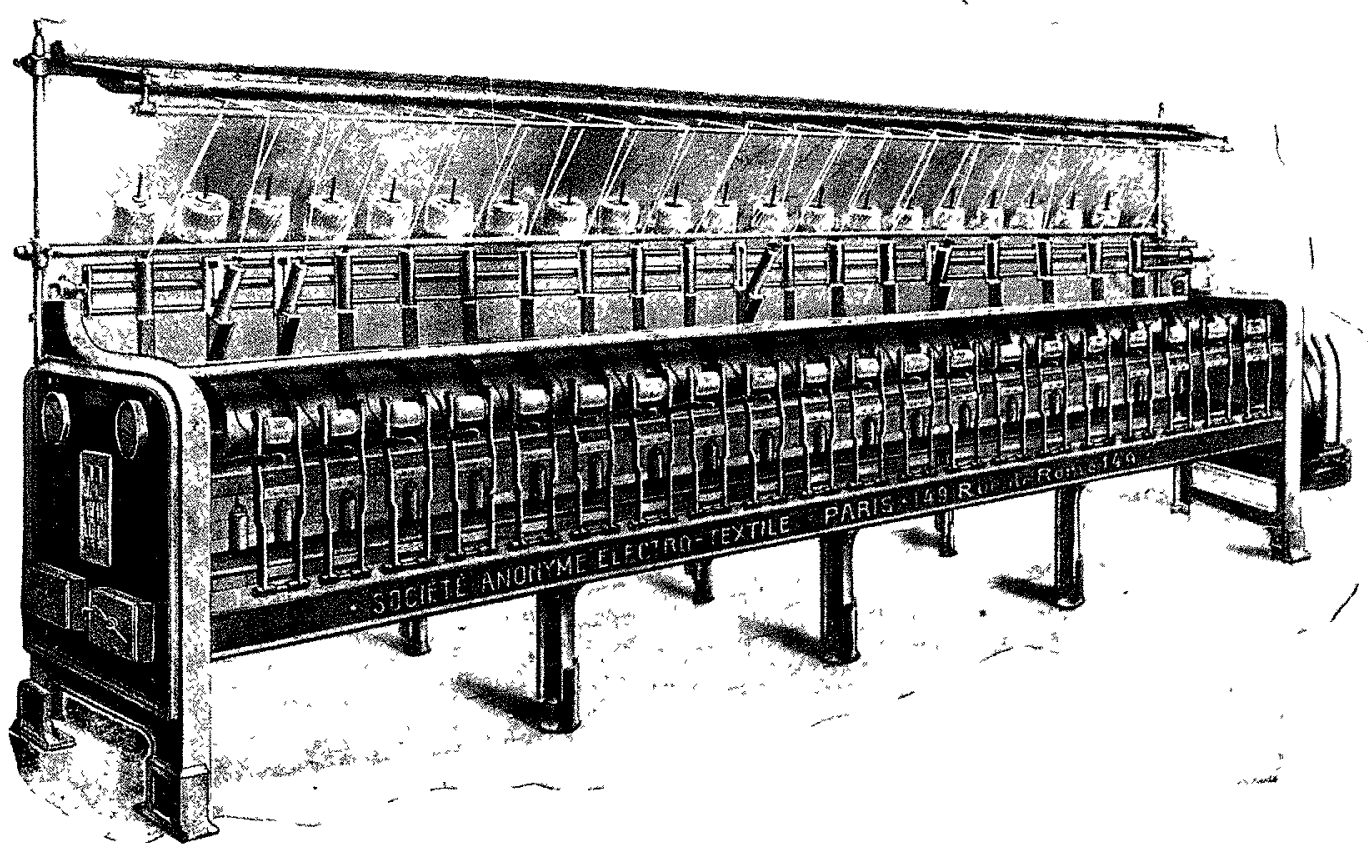

FIG. 1. - Vue d'un métier à gazer. 
Le collecteur de la ventilation aspirante $a$ porte, en regard de chaque brûleur, une tubulure sur laquelle vient se fixer la boîte d'aspiration $c$. Cette boîte se compose d'uné colonnette conique, creuse, en métal, dont la partie inférieur s'ajuste sur la tubulure, tandis que la partie supérieure du cône se raccorde avec la base de l'appareil électrique de gazage, sans aucune aspérité intérieure, et suivant une forme étudiée de manière à réduire le plus possible les pertes de charge éprouvées par l'air de la ventilation: le maximum d'effet utile est ainsi obtenu, et l'on évite toute accumulation de poussière.

La boîte d'aspiration est fermée en avant par un couvercle à charnière $f$, articul í en $g$, et portant à sa partie inférieure un bras de levier $h$ qui forme contrepoids. Ce contrepoids est réglé de telle façon que le couvercle puisse tomber de lui-même, suivant le tracé ponctué de la figure 3 . Ce couvercle, plat jusqu'à l'arasement, forme un demianneau en regard de l'appareil électrique de gazage, butant contre la base de l'appareil gazeur, et fermant tout l'ensemble aussi hermétiquement qu'll convient.

La dépression produite par le ventilareur aspirant dans le collecteur $a$ se manifeste dans la boîte $c$ par un appel d'air qui entre par la partie supérieure ouverte de la boîte en $j$, et vient lécher le fil sur toute la longueur soumise à l'incandescence du tube brûleur; toutes les parties à flamber sont ainsi soumises à une combustion complète. Deux ouvertures $k$, ménagées dans le couvercle, permettent de bien suivre la trajectoire du fil dans le tube.

Le couvercle porte à sa partie supérieure un crochet $l$ qui sert au guidage du fil. Ce fil vient du râtelier, et est amenć à l'appareil gazeur lorsque le couvercle occupe la position indiquée par le tracé ponctué de la figure 5 , et le bras de levier repose sur la partie plate $m$ du levier $n$ de commande du lève-fil.

Le fil passe en I, ensuite dans le guide-racleur $o$ et, par un mouvement de gauche à droite, se trouve engagé dans la fente $p$ du couvercle; de là, il s'enroule sur une bobine d'appel en passant dans la fente du tambour d'entraine-

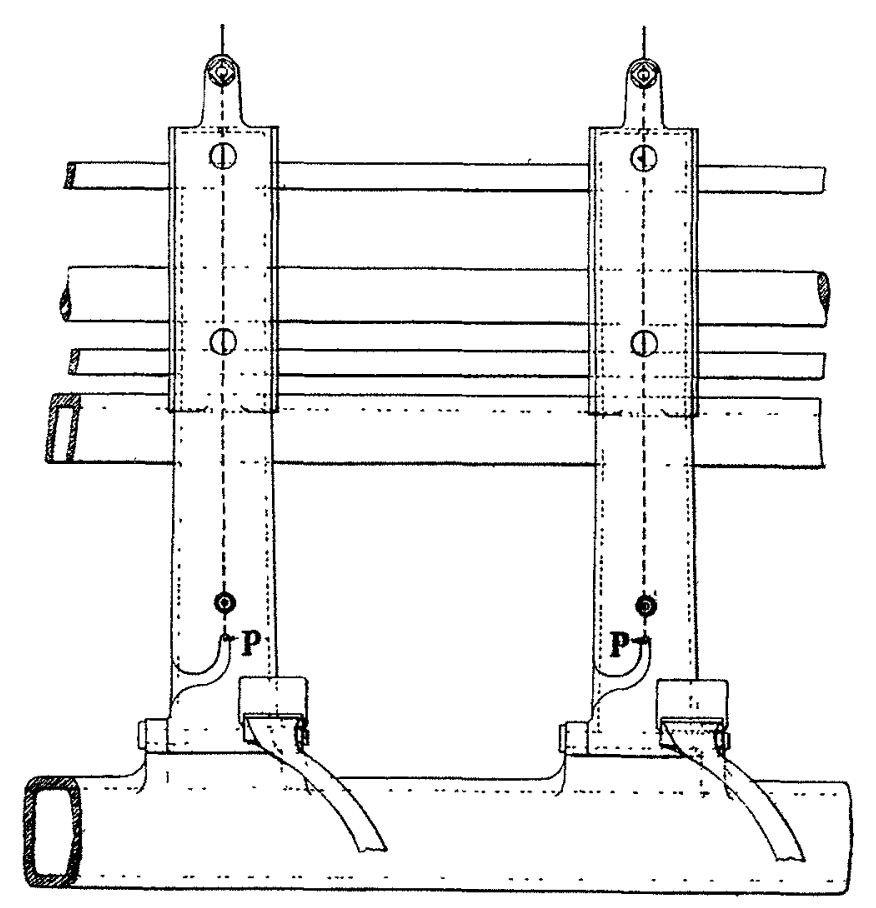

ment (la bobine et le tambour ne sont pas représentés sur les figures 4 et 5 ):

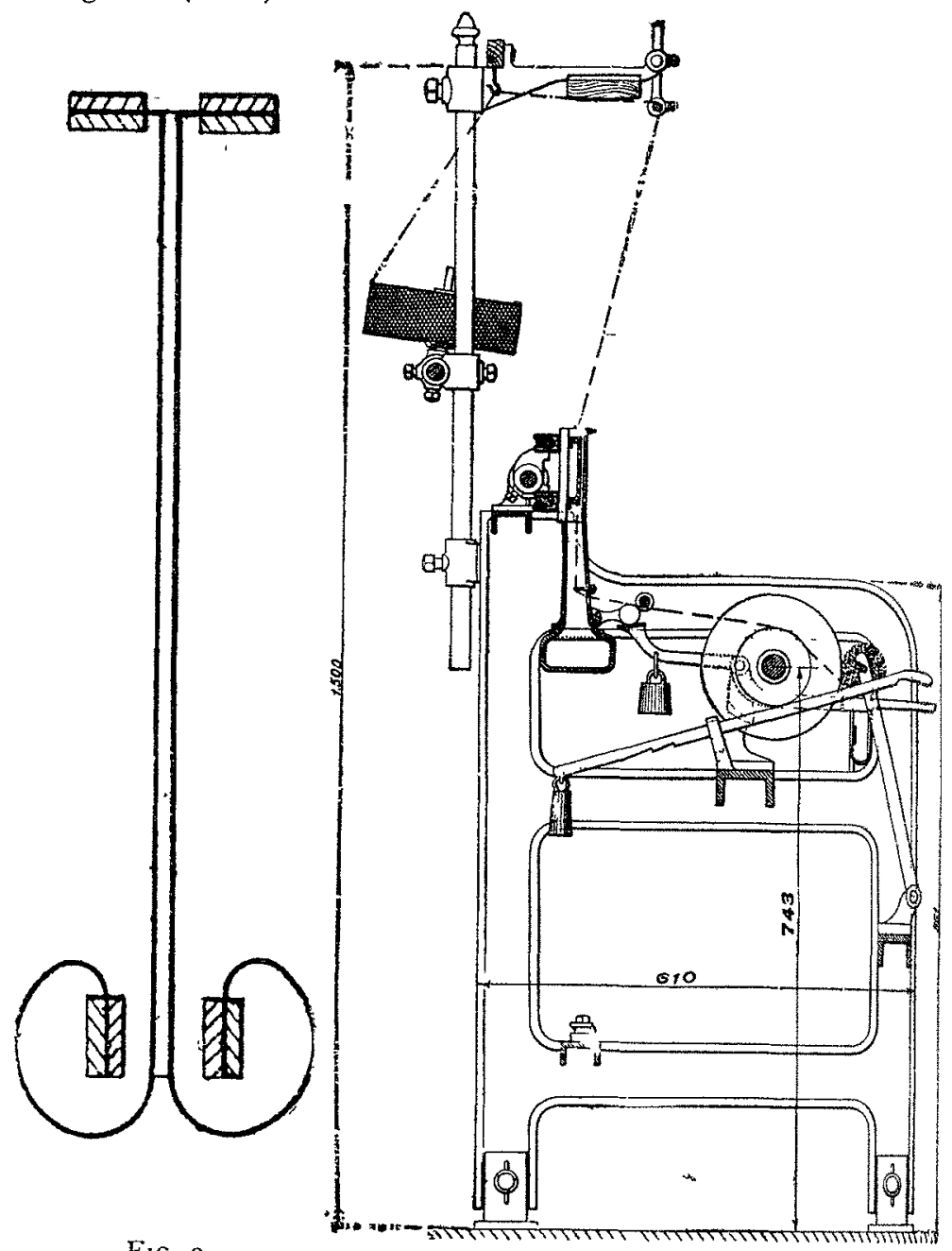

Fig. 2.

Tube gazeur.

Fig. 3. - Machine à gazer.

Pour faire pénétrer le fil dans le brûleur, il suffit d'appuyer sur l'extrémité du levier $n$, dont la partie plate, qui se

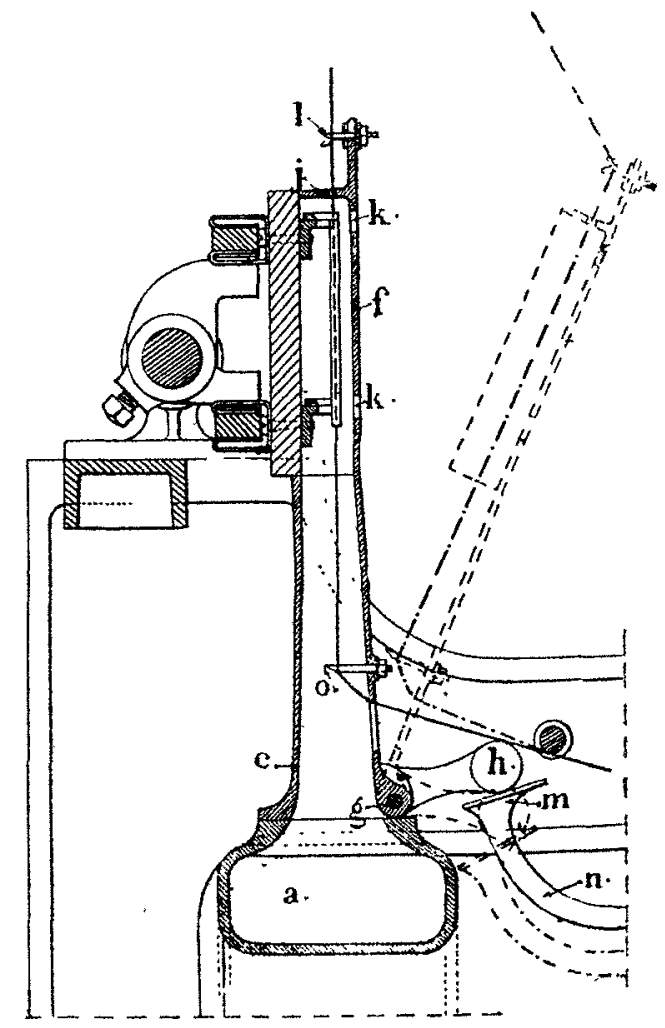

FIG. 4 et 5. - Elévation et coupe du système gazeur. 
trouve dans la position indiquée par le tracé en éléments de la figure 5, vient prendre aussitôt celle qui est indiquée par le tracé plein. Dans ce mouvement, $h$ est soulevé, et le couvercle se referme complètement, entrainant avec lui le fil qui se loge dans le tube en même temps que commence le dévidement. Le levier $h$ est maintenu dans cette position al'aide d'un cran d'arrêt monté sur ressort.

Pour sortir le fil du tube gazeur, il suffit de déclancher le ressort avec le doigt, et le levier $n$, entrainé, par un contrepoids, retombe en même temps que le couvercle, et reprend la position représentée par le tracé en éléments de la figure 5 . rhéostat absorbant l'énergie en excès, mais, si ce dispositif réduit au minimum les frais de premier établissement, il est peu économique au point de vue de l'exploitation; aussi est-il plus avantageux d'alimenter les métiers au moyen d'une sorte de commutatrice, composée d'une dynamo shunt ordinaire à laquelle on ajoute deux balais supplémentaires reliés au métier. En décalant ces balais par rapport à ceux qui sont branchés sur le réseau, on obtient toute la gamme des voltages, depuis zéro jusqu’à celui du réseau $\left(^{\star}\right)$.

Dans le cas de courant alternatif, on emploie simplement un transformateur statique $T$, dont l'enroulement secondaire est.muni d'un commutateur à plots $\mathrm{R}$, permettant de

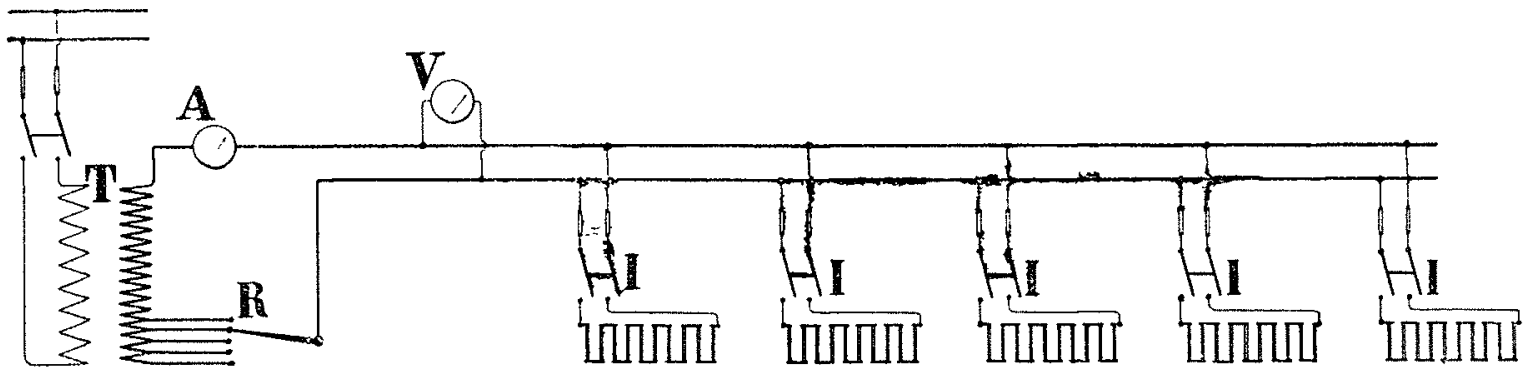

FIG. 0. - Schéma du montage électrique.

Les poussières et résidus de la combustion sont détachés par le passage du fil sur le guide-racleur o, lequel suffit pour certains genres de fils, et que l'on complète, s'il est nécessaire, par des petites galets fixes, ou mobiles sur leurs axes placés en travers de la boîte d'aspiration.

l'évacuation des poussières et des produits gazeux de la combustion des filaments textiles est ici parfaitement assurée dans les conditions prescrites par l'article $6 \mathrm{du}$ décret du 29 novembre igo4. On voit que le système de ventilation adopté n'est autre chose qu'une ventilation partielle ou localisée du type per descensum.

Cette ventilation permet d'obtenir avec les métiers à gazer électriques une amélioration considérable des conditions de travail des ouvrières. De plus, les celles-cin'ont pas la vue qui se fatigue par un rayonnement intensif d'une source calorifique, et chaque gazeuse peut conduire un métier avec la plus grande facilité.

Les brûleurs sont montés sur un bobinoir sur lequel s'effectuent simultanément le dévidement continu du fil à gazer, le gazage, et le rebobinage du fil gazé.

Après avoir traversé le brûleur, le fil, convenablement guidé, s'engage dans la fente hélicoïdale d'un tambour animé d'un mouvement de rotation. Le passage du fil dans cette fente lui imprime un mouvement alternatif de translation qui permet de l'enrouler régulièrement sur une bobine, dans les conditions les plus propres à prévenir tout éboulement. Cette bobine est maintenue en contact avec in poulie hélicordale, qui l'entraîne et lui communique un mouvement de rotation permettant le bobinage à vitesse périphérique constante.

Les appareils de réglage et de mesure sont placés sur un tábleau à la portée de l'ouvrière qui a ainsi sous la main tous les moyens de conduite de son métier.

Les brûleurs électriques fonctionnent sous une tension de 2 à 5 volts, en absorbant un courant de 20 à 35 ampères suivant le genre de filé que l'on a à gazer.

Lorsque les métiers à gazer doivent être branchés sur un réseau de distribution où le voltage est fixe, il faut employer divers dispositifs régulateurs de tension.

Dans le cas du courant continu, on peut employer un faire varier le rapport de transformation; on réunit alors en série un certain nombre de brûleurs, de manière à constituer un groupe I (fig. 6), et tous les groupes semblables sont branchés en parallèle sur le circuit secondaire.

M. P.

\section{La Fabrication des Électrodes}

\section{(Suite)}

\section{II. - MATIÈRES PREMIÈRES EMPLOYÉES DANS LA FABRICATION DES ÉLECTRODES}

Les charbons artificiels employés dans la fabricalion des électrodes sont :
A. Le charbon de cornue;
$B$. Le coke de pétrole;
C. Le coke de brai;

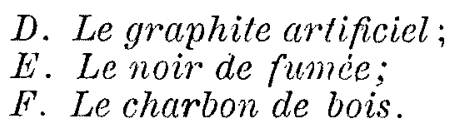

A. - Gharbon de cornue. - Le charbon de cornue est celui qui se dépose sur les parois intérieures des cornues à gaz. Il provient de la décomposition d'une partie des hydrocarbures fournis par la distillation pyrogénée de la houille et qui, au contact des parois de la cornue chauffées au rougc, se dissocient en laissant un résidu de carbone.

L'aspectplysique du charbon de cornue est très variable. C'est ainsi qu'on a des échantillons noirs, compacts, excessivement durs, sans aucunestructure particuliere, dont quel. ques-uns ont gardé la forme incurvée de la cornue. D'autres charbons de cornue présentent une structure feuilletée comme de l'ardoise, très facilement clivable, ot tachent les doigts. Nous avons vu des échantillons présentant l'apparence de curieuses stalactites et d'autres qui, au licu d'étre noir, étaient d'un blanc métallique argenté du plus bel effet. Ces derniers types sont très friables, et aussi très purs, ils ont moins de $1 \%$ de cendres. Les variétés feuilletces et en forme de stalactites sont également très pures et les cendros y varient de 1 à $1,7 \%$. Le charloon de cornue de la

(*) Dans le cas où l'on désire produire soi-même son électricité, l'Electro-Textile préconise l'emplo1 d'une dynamo-génératrice donnant toutes les tensions correspondant aux divers régimes, sans crachement des balais, par simple réglage de l'excitation réalisé grâce au déplacement d'un troisième halais supplémentairc. 